\title{
Superior cerebellar artery aneurysms: incidence, clinical presentation and midterm outcome of endovascular treatment
}

\author{
Jo P. P. Peluso • Willem Jan van Rooij • \\ Menno Sluzewski • Guus N. Beute
}

Received: 6 February 2007 / Accepted: 3 May 2007 / Published online: 4 July 2007

(C) Springer-Verlag 2007

\begin{abstract}
Introduction The aim of this retrospective study was to determine the incidence, clinical presentation and midterm clinical and imaging outcome of endovascular treatment of 34 superior cerebellar artery (SCA) aneurysms in 33 patients. Methods Between January 1995 and January 2007, 2,112 aneurysms were treated in our institution, and 36 aneurysms in 35 patients were located on the SCA (incidence $1.7 \%$ ). Two of three distal SCA aneurysms were excluded. All the remaining 34 SCA aneurysms, of which $22(65 \%)$ were ruptured and $12(35 \%)$ were unruptured, in 33 patients were treated by endovascular techniques. There were 6 men and 27 women ranging from $29-72$ years. In 14 patients $(42 \%)$ multiple aneurysms were present.

Results Initial angiographic occlusion was (near) complete in 32 aneurysms (94\%) and incomplete in 2 aneurysms (6\%). Complications leading to permanent morbidity or death occurred in two patients $(6.1 \%, 95 \%$ CI 0.6 to $20.60 \%$ ). Outcome at 6 months follow-up in 31 surviving patients was GOS5 in $26(84 \%)$, GOS4 in $4(13 \%)$ and GOS3 in 1 patient (3\%). There were no episodes of (re) bleeding during 118 patient-years of follow-up. The 6month angiographic follow up in 28 SCA aneurysms and extended angiographic follow-up in 19 showed stable occlusion in 27 aneurysms. No additional treatments were performed.
\end{abstract}

J. P. P. Peluso · W. J. van Rooij $(\bowtie) \cdot$ M. Sluzewski

Department of Radiology, St. Elisabeth Ziekenhuis,

Hilvarenbeekseweg 60,

5022 GC Tilburg, The Netherlands

e-mail: radiol@knmg.nl

G. N. Beute

Department of Neurosurgery, St. Elisabeth Ziekenhuis,

Tilburg, The Netherlands
Conclusion SCA aneurysms are rare with an incidence of $1.7 \%$ of treated aneurysms at our institution. They are frequently associated with other aneurysms. Endovascular treatment is effective and safe in excluding the aneurysms from the circulation.

Keywords Superior cerebellar artery - Aneurysm . Coiling $\cdot$ Outcome

\section{Introduction}

Aneurysms located on the superior cerebellar artery (SCA) are uncommon and their presentation, natural history and clinical management are poorly understood $[1,2]$. Reports about the endovascular or surgical management of SCA aneurysms are rare and are usually incorporated in clinical series of basilar artery or posterior circulation aneurysms [3-18].

The aim of this retrospective study was to determine the incidence, clinical presentation and midterm clinical and imaging results of endovascular treatment of 35 patients with 36 SCA aneurysms.

\section{Methods}

Patients

Between January 1995 and January 2007, 2,112 aneurysms were treated in our institution. Of the 2,112 treated aneurysms, $324(15 \%)$ were located in the posterior circulation and $1,788(85 \%)$ in the anterior circulation. Surgery was performed in 970 aneurysms and endovascular treatment in 1,142 aneurysms. Of the 2,112 treated 


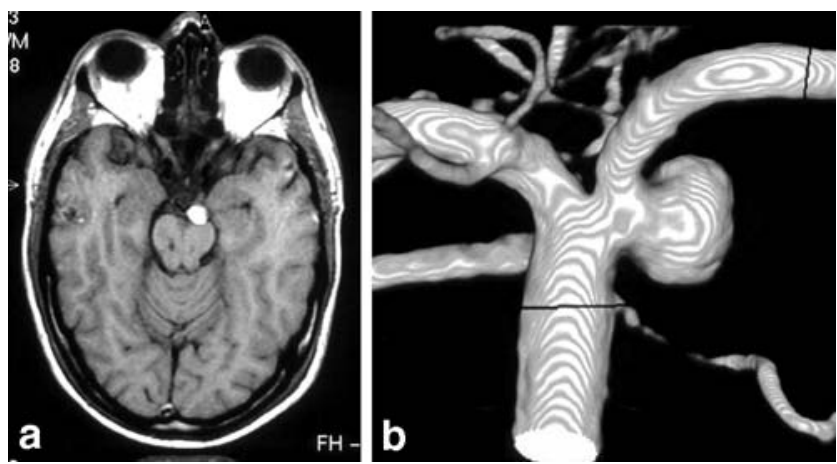

Fig. 1 Incidentally found SCA aneurysm in a 39-year-old man with vertebrobasilar embolic infarcts. a T1-weighted MR image shows a hyperintense lesion adjacent to the brainstem. b 3-D vertebral angiogram reveals a small SCA aneurysm

aneurysms, 36 aneurysms in 35 patients were located on the SCA, resulting in an incidence of $1.7 \%$ of all treated intracranial aneurysms and $11.1 \%$ of treated posterior circulation aneurysms. We excluded one patient with a peripheral SCA flow aneurysm associated with an arteriovenous malformation and one peripheral partially thrombosed SCA aneurysm presenting with trochlear nerve palsy. In one patient with four aneurysms, one aneurysm was located on the proximal right SCA and one aneurysm on the distal left SCA. All 33 proximal SCA aneurysms were primarily based on the basilar artery at the branching point of the SCA with or without incorporation of the SCA origin in the base. All 34 SCA aneurysms in 33 patients were treated by endovascular techniques. There were 6 men and 27 women with a mean age of 50.7 years (median 49 years, range 29 72 years). Of 34 aneurysms, 22 (65\%) had ruptured, 7 $(20 \%)$ were additional to another ruptured aneurysm, 4 (12\%) presented with symptoms of a mass effect (trigeminal neuralgia 2, oculomotor palsy 2 ) and one (3\%) was an incidental finding (Fig. 1). The mean size of the 34 SCA aneurysms was $7.3 \mathrm{~mm}$ (median $6 \mathrm{~mm}$, range 2-27 mm).

The clinical condition of the 22 patients with a ruptured SCA aneurysm at the time of treatment was HH I-II in 16, HH III in 3 and HH IV-V in 3. Seven SCA aneurysms in six patients were additional to another ruptured aneurysm

Fig. 2 Pre- and posttreatment images of two patients with multiple posterior circulation aneurysms. a A 44-year-old man with a ruptured middle cerebral artery aneurysm and four additional aneurysms. 3-D vertebral angiogram reveals right SCA aneurysm (short single arrow), left distal SCA aneurysm (long single arrow) and basilar tip aneurysm (pair of arrows). b Complete occlusion after coiling. c A 61-year-old woman with a ruptured middle cerebral artery aneurysm and additional aneurysms on the left SCA and basilar tip. d Adequate occlusion of basilar tip and SCA aneurysms
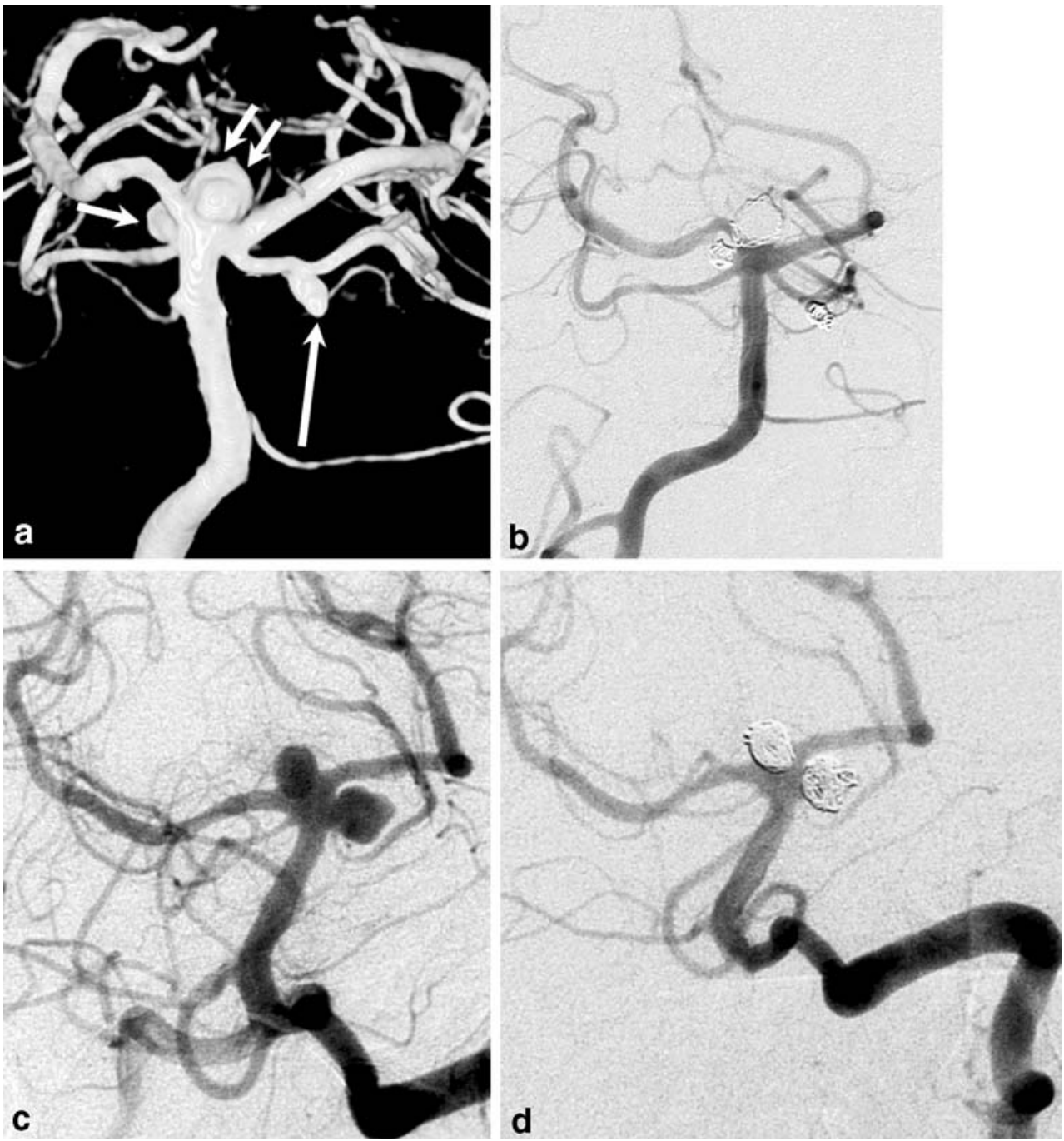
and were coiled in the same session as the ruptured aneurysm. The clinical condition of these six patients was $\mathrm{HH}$ I-II in five and HH IV-V in one.

Of the 33 patients, $14(42 \%)$ had multiple aneurysms (Fig. 2) for a total of 72 aneurysms: 5 patients had 2 aneurysms, 3 patients had 3 aneurysms, 3 patients had 4 aneurysms, 1 patient had 5 aneurysms, 1 patient had 7 aneurysms and 1 patient had 8 aneurysms. Of the 72 aneurysms in 35 patients, 53 were coiled, 13 were clipped, and 6 were small $(<2 \mathrm{~mm})$ and left untreated.

\section{Coiling procedure}

Coiling of aneurysms was performed on a biplane angiographic unit (Integris BN 3000 Neuro, Philips Medical Systems, Best, The Netherlands) with the patient under general anaesthesia and systemic heparinization. Heparin was continued intravenously or subcutaneously for $48 \mathrm{~h}$ after the procedure, followed by low-dose aspirin for 3 months orally. Coiling was performed with Guglielmi detachable coils (GDC, Boston Scientific, Fremont, Calif.) or TruFill DCS coils (Cordis, Miami, Fl.). The aim of coiling was to pack the aneurysm as densely as possible, until not a single additional coil could be placed. Four wide-necked aneurysms $(12 \%)$ were coiled with the aid of a supporting balloon in the basilar artery. One aneurysm was located distal on the SCA and was selectively occluded with coils sparing the parent SCA. Complications of coiling were recorded.

Initial angiographic results of coiling were classified as complete occlusion $(100 \%)$, near complete occlusion $(90$ $100 \%)$ and incomplete occlusion $(<90 \%)$.

\section{Clinical and angiographic follow-up}

Patients who survived the hospital admission period were scheduled for a follow-up visit in the outpatient clinic 6 weeks after treatment and for follow-up angiography
Fig. 3 Ischemic complication of coiling in a 71-year-old woman with a ruptured SCA aneurysm. a Vertebral angiogram demonstrates a widenecked right SCA aneurysm with the SCA arising from the sac. b, c Balloon-assisted coiling with adequate occlusion and preserved flow in the SCA. The patient did not awake from general anaesthesia. Immediate control angiography revealed that the SCA had become occluded (not shown). d MRI several days later showing brainstem and cerebellum infarctions in the SCA territory
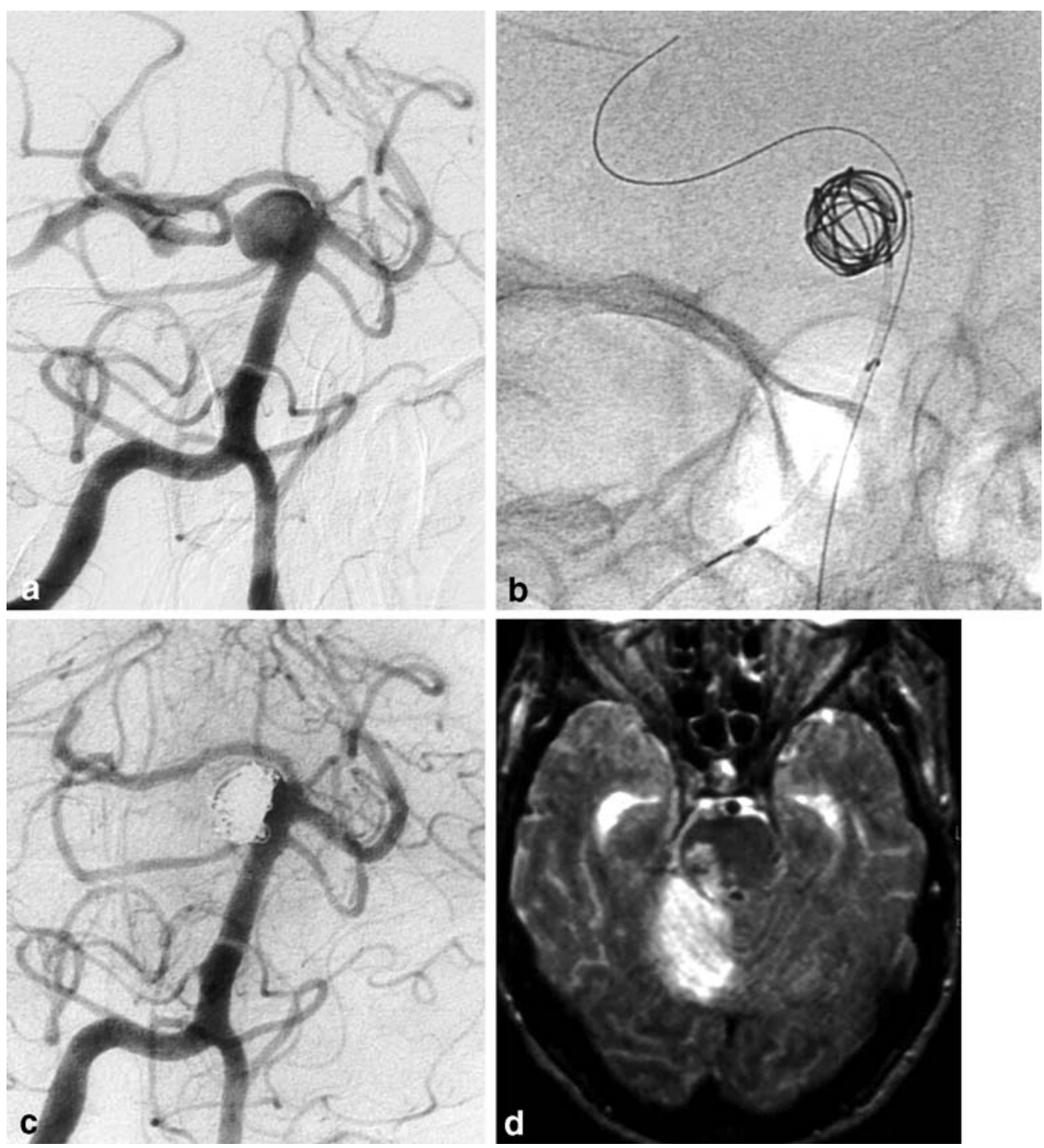
after 6 and 18 months. Neurological status according to the Glasgow Outcome Scale (GOS) was evaluated at every outpatient clinic visit and at every admission for follow-up angiography. The results of follow-up angiography were classified in the same way as for initial angiographic results.

\section{Results}

Initial angiographic results and complications

Initial angiographic occlusion was complete or near complete $(90-100 \%$ occlusion) in 32 aneurysms and incomplete in 2 aneurysms (both $80 \%$ occlusion). Complications leading to permanent morbidity or death occurred in two patients. The first patient was a 42-year-old woman who presented with a ruptured 2-mm wide-necked SCA aneurysm in HH I. During insertion of a coil with the aid of a supporting balloon, a rupture occurred and the patient died $12 \mathrm{~h}$ later (mortality 1 of 33, 3.0\%, 95\% CI $0.01-$ $16.65 \%$ ). The second patient was a 71-year-old woman admitted with a ruptured $10-\mathrm{mm}$ wide-necked SCA aneurysm (Fig. 3). The SCA itself originated from the sac. The aneurysm was coiled with the aid of a supporting balloon with adequate occlusion and preserved flow in the SCA. However, the patient did not awake from general anaesthesia. Immediate control angiography revealed that the SCA had become occluded. MRI several days later showed infarctions in the vascular territory of the SCA in the brainstem and cerebellum. The patient gradually recovered but remained dependent. At the time of this report almost 4 years later she was still in a nursing home (procedural morbidity 1 of $33,3.0 \%, 95 \%$ CI $0.01-$ $16.65 \%)$.

\section{Clinical follow-up}

Clinical follow-up was available for all 33 patients. One patient died of a procedural rupture and another patient died of diffuse vasospasm after subarachnoid haemorrhage (SAH). The remaining 31 patients had clinical follow-up of mean 45 months (median 44.5 months, range 4103 months, 118 patient-years). Outcome at 6 months follow up of these 31 patients was GOS 5 in $26(84 \%)$, GOS 4 in $4(13 \%)$ and GOS 3 in 1 patient (3\%). Symptoms of mass effect on cranial nerves in four patients were cured in all. During extended follow up, two patients died of unrelated disease 3 and 6 years after coiling of a SCA aneurysm: pulmonary embolism in one patient and disseminated bronchial carcinoma in the other patient. There were no episodes of (re)bleeding during the 118 patient-years of follow-up.
Angiographic follow-up

Of the 31 surviving patients, 4 refused follow-up angiography. The remaining 27 patients (with 28 SCA aneurysms) all had 6 months follow-up angiography and 19 had extended angiographic follow-up of a mean of 31 months (median 22 months, range 18-84 months). Stable complete or near-complete occlusion was apparent in 25 of 28 aneurysms. In one patient, a 66-year-old man with a 27-mm partially thrombosed SCA aneurysm presenting with trigeminal neuralgia, the coils had migrated into the intraluminal thrombus at the 6-month follow-up angiography. However, the trigeminal neuralgia was cured and the aneurysm was not retreated. The patient died 6 years later of disseminated bronchial carcinoma. Two aneurysms in two patients that were initially incompletely occluded (both $80 \%$ occlusion) remained so at extended angiographic follow-up. Additional treatment was judged impossible in both patients.

\section{Discussion}

In this study, we found that SCA aneurysms are rare with an incidence of $1.7 \%$ of all treated intracranial aneurysms and $11.1 \%$ of treated posterior circulation aneurysms at our institution. The majority of patients presented with SAH and $42 \%$ of patients also had aneurysms at other locations. This frequent association with multiple aneurysms has not been established before. Endovascular occlusion with detachable coils is feasible with good angiographic and clinical results and with low morbidity [3].

The proximal SCA has an intimate relation with cranial nerves III, IV and V [19]. Aneurysms on the SCA may therefore present with palsies of these nerves, as was the case in four of our patients [10, 11]. After coiling, symptoms of nerve compression regressed in all four patients.

One of 34 SCA aneurysms was located distally on the artery. It was possible to occlude this aneurysm with sparing of the parent SCA. When selective occlusion of such an aneurysm is not possible, simultaneous occlusion of the aneurysm together with the parent artery can be performed. Collateral blood flow may limit ischemic infarctions and outcome is usually good [4, 12-15].

Although surgical treatment of SCA aneurysms in experienced hands is associated with good results, access may be challenging needing destructive approaches [2022]. The presence of important perforating arteries and adjacent cranial nerves makes clipping of SCA aneurysms difficult and complications may occur. Because SCA aneurysms are rare, not many surgeons will gain experience in the treatment of these aneurysms. In addition, many 
patients are in bad clinical condition and are not good surgical candidates in the acute phase.

While surgery for SCA aneurysms is often difficult, limited to good-grade patients and associated with substantial morbidity, coiling is technically easy and can also be performed in the acute phase of haemorrhage in bad-grade patients. This makes coiling the method of choice to treat SCA aneurysms.

\section{Conclusion}

SCA aneurysms are rare with an incidence of $1.7 \%$ of treated aneurysms at our institution. There is a frequent association with aneurysms at other locations. Most patients present with SAH. Since the SCA is closely related to cranial nerves III, IV and V, some aneurysms present with palsies of these nerves. Endovascular occlusion with detachable coils is feasible with good angiographic and clinical results.

Conflict of interest statement We declare that we have no conflict of interest.

\section{References}

1. Locksley HB (1966) Natural history of subarachnoid hemorrhage, intracranial aneurysms and arteriovenous malformations. Based on 6368 cases in the cooperative study. J Neurosurg 25:219-239

2. Lubicz B, Leclerc X, Gauvrit JY, Lejeune JP, Pruvo JP (2003) Endovascular treatment of peripheral cerebellar artery aneurysms. AJNR Am J Neuroradiol 24:1208-1213

3. Haw C, Willinsky R, Agid R, TerBrugge K (2004) The endovascular management of superior cerebellar artery aneurysms. Can J Neurol Sci 31:53-57

4. Pierot L, Boulin A, Castaings L, Rey A, Moret J (1996) Selective occlusion of basilar artery aneurysms using controlled detachable coils: report of 35 cases. Neurosurgery 38:948-953

5. Lempert TE, Malek AM, Halbach VV, Phatouros CC, Meyers PM, Dowd CF, Higashida RT (2000) Endovascular treatment of ruptured posterior circulation cerebral aneurysms. Clinical and angiographic outcomes. Stroke 31:100-110
6. Uda K, Murayama Y, Gobin YP, Duckwiler GR, Vinuela F (2001) Endovascular treatment of basilar artery trunk aneurysms with Guglielmi detachable coils: clinical experience with 41 aneurysms in 39 patients. J Neurosurg 95:624-632

7. Ushikoshi S, Kikuchi Y, Houkin K, Miyasaka K, Abe H (1999) Aggravation of brainstem symptoms caused by a large superior cerebellar artery aneurysm after embolization by Guglielmi detachable coils - case report. Neurol Med Chir (Tokyo) 39:524-529

8. Sagoh M, Hirose Y, Murakami H, Mayanagi K (1997) The outcome of early surgical management of ruptured posterior circulation aneurysms. Neurol Res 19:385-388

9. Nagasawa S, Kobata H, Aoki J, Kawanishi M, Ohta T (1996) A large thrombosed superior cerebellar artery aneurysm: a case report. Surg Neurol 45:36-38

10. Agostinis C, Caverni L, Moschini L, Rottoli MR, Foresti C (1992) Paralysis of fourth cranial nerve due to superior-cerebellar artery aneurysm. Neurology 42:457-458

11. Collins TE, Mehalic TF, White TK, Pezzuti RT (1992) Trochlear nerve palsy as the sole initial sign of an aneurysm of the superior cerebellar artery. Neurosurgery 30:258-261

12. Chaloupka J, Putman C, Awad I (1996) Endovascular therapeutic approach to peripheral aneurysms of the superior cerebellar artery. AJNR Am J Neuroradiol 17:1338-1442

13. Cloft H, Kallmes D, Jensen M, Lanzino G, Dion J (1999) Endovascular treatment of ruptured, peripheral cerebral aneurysms: parent artery occlusion with short Guglielmi detachable coils. AJNR Am J Neuroradiol 20:308-310

14. Cognard C, Weill A, Tovi M, Castaings L, Rey A, Moret J (1999) Treatment of distal aneurysms of the cerebellar arteries by intraaneurysmal injection of glue. AJNR Am J Neuroradiol 20:780-784

15. Eckard D, O’Boynick P, McPherson C et al (2000) Coil occlusion of the parent artery for treatment of symptomatic peripheral intracranial aneurysms. AJNR Am J Neuroradiol 21:137-142

16. Danet M, Raymond J, Roy D (2001) Distal superior cerebellar artery aneurysm presenting with cerebellar infarction: report of two cases. AJNR Am J Neuroradiol 22:717-720

17. Mabuchi S, Kamiyama H, Abe H (1992) Distal aneurysms of the superior cerebellar artery and posterior inferior cerebellar artery feeding an associated arteriovenous malformation: case report. Neurosurgery 30:284-287

18. Gacs G, Vinuela F, Fox A, Drake C (1983) Peripheral aneurysms of the cerebellar arteries: review of 16 cases. J Neurosurg 58:63-68

19. Rhoton AL Jr (2000) The cerebellar arteries. Neurosurgery 47 (3 Suppl):S29-S68

20. Gonzalez LF, Amin-Hanjani S, Bambakidis NC, Spetzler RF (2005) Skull base approaches to the basilar artery. Neurosurg Focus 19:E3

21. Kawase T, Bertalanffy H, Otani M, Shiobara R, Toya S (1996) Surgical approaches for vertebro-basilar trunk aneurysms located in the midline. Acta Neurochir (Wien) 138:402-410

22. Seifert V, Stolke D (1996) Posterior transpetrosal approach to aneurysms of the basilar trunk and vertebrobasilar junction. J Neurosurg 85:373-379 